\title{
03 HELMET USE AMONG MOTORCYCLISTS IN CAMBODIA: A SURVEY OF USE, KNOWLEDGE, ATTITUDES, AND PRACTICES
}

doi:10.1136/injuryprev-2012-040590s.3

${ }^{1} \mathrm{AM}$ Bachani, ${ }^{2} \mathrm{~S}$ Sann, ${ }^{1} \mathrm{C}$ Zogg, ${ }^{3} \mathrm{M}$ Ballesteros, ${ }^{2} \mathrm{C}$ Ear, ${ }^{1} \mathrm{AA}$ Hyder. ${ }^{1} \mathrm{~J} o h n s$ Hopkins International Injury Research Unit, Health Systems Program, Department of International Health, Johns Hopkins Bloomberg School of Public Health, Baltimore, MD, USA; ${ }^{2}$ Handicap International Belgium, Phnom Penh, Cambodia; ${ }^{3}$ Centers for Disease Control and Prevention, Atlanta, GA, USA

Background Motorcycle-related road traffic injuries are a significant contributor to the burden of disease in low- and middle-income countries. To reduce this risk, helmet use has been proven an effective method.

Aims/Objective/Purpose Given an increase in motorcycle use in Cambodia, this study aims to assess the current status of helmet use, as well as the knowledge, attitudes, and practices of use among motorcyclists.

Methods Helmet observations were conducted in Phnom Penh, Kandal, Kampong Speu, Siem Reap, and Kampong Cham to assess the current status of helmet use. In addition, roadside knowledge, attitudes, and practice interviews were also conducted in Phnom Penh, Kandal, and Kampong Speu to determine prevailing beliefs.

Results/Outcomes Though awareness of the benefits of wearing a helmet is high, actual helmet use remains low. Daytime usage is higher than nighttime (45\% vs $32 \%$ across all study sites in January 2012), and these proportions are significantly higher among drivers than among passengers (ratio of $8.2: 1.0$ ). Among those interviewed in 2011, 55\% said that use of a helmet depends on where they drive. The top three reasons for always wearing a helmet included: lifesaving potential, legal duty, and police fines. Helmet quality, price, style, and colour were also important factors influencing the decision to purchase a helmet.

Significance/Contribution to the Field The results of this study will be used to assist with better planning and implementation of injury prevention strategies and to address the continuing need to improve the proportion of all-day helmet wearing, especially at night and among motorcycle passengers in Cambodia. 